\title{
New potential super-incompressible phase of $\operatorname{ReN}_{2}$
}

\author{
Yanling Li $^{1,2,3}$, Zhi Zeng ${ }^{1}$ \\ 1.Key Laboratory of Materials Physics, \\ Institute of Solid State Physics, Chinese Academy of Sciences, \\ Hefei 230031,People's Republic of China \\ 2.Department of physics, Xuzhou Normal University, \\ Xuzhou 221116, People's Republic of China \\ 3. Graduate School of the Chinese Academy of Sciences, \\ Beijing 100049, People's Republic of China
}

(Dated: July 15, 2018)

\begin{abstract}
The structural, elastic, and electronic properties of $\mathrm{ReN}_{2}$ are investigated by first-principles calculations with density functional theory. The obtained orthorhombic Pbcn structure is energetically the most stable structure at ambient pressure. $\mathrm{ReN}_{2}$ is a metallic, superincompressible solid and presents a rather elastic anisotropy. The estimated Debye temperature and hardness are 735 $\mathrm{K}$ and $17.1 \mathrm{GPa}$, respectively. Its estimated hardness is comparative to that of $\mathrm{Si}_{3} \mathrm{~N}_{4}$.

PACS numbers: 61.50.Ks, 62.20.-x, 71.15.Mb, 71.20.-b, 71.20.Be
\end{abstract}




\section{INTRODUCTION}

Transition metal nitrides are of great technological and fundamental importance because of their strength and durability as well as their useful optical, electronic, and magnetic properties. [1] By incorporating B, C, N, or O atoms into the interstitial sites of transition metals with highly valence electron density (VED), the compounds of transition metal with highly hardness and strong incompressibility can be synthesized by means of high pressure experiments. [2, 3] For these reasons, most recently, three transition metal dinitrides, $\mathrm{PtN}_{2}$, , 1] $\mathrm{IrN}_{2}$, [4] and $\mathrm{OsN}_{2}$, [4] have been successfully synthesized under extreme conditions of pressure and temperature. Very large bulk moduli observed in experiments make them be candidates of superhard materials, which could be used in cutting tools and wear-resistant coatings. Meanwhile, many theoretical investigations have been performed to explore their structures, for that the crystal structure is an important prerequisite of understanding their physical properties. The search for the structural forms of $\mathrm{PtN}_{2}, \mathrm{OsN}_{2}$ and $\mathrm{IrN}_{2}$ with the lowest energy and mechanical stability usually considers hypothetic structures of orthorhombic, tetragonal, hexagonal and cubic crystal lattice. The structures of $\mathrm{PtN}_{2}$ and $\mathrm{OsN}_{2}$ are indexed as cubic (pyrite structure, $P a \overline{3})[1$, 5] and orthorhombic (marcasite structure, Pnnm). [6, , 7, 8] While it is very difficult to determine the structure of $\mathrm{IrN}_{2}$ in experiment because of the large intensity ratio (1:100) of X-ray diffraction peaks between nitride and pure metal. The firstprinciples calculations have shown that $\mathrm{IrN}_{2}$ is monoclinic $\left(\mathrm{CoSb}_{2}\right.$ structure, $\left.P 2_{1} / c\right)$. [8] As a neighbor of noble transition metal Os, the diboride of Re has been successfully synthesized, [3], 9] however the dinitride of Re to date has not been reported experimentally. Theoretically, Zhao et al suggested that $\mathrm{ReN}_{2}$ with $\mathrm{P}_{2} / \mathrm{mnm}$ structure is thermodynamically stable at ambient conditions and up to $76 \mathrm{GPa}$.[10] Here, we readdress structural and elastic properties of $\mathrm{ReN}_{2}$ and find that a new phase with Pbcn symmetry is more stable than that with $P 4_{2} / m n m$ symmetry.

\section{COMPUTATIONAL DETAILS}

All calculations are performed by the CASTEP code[11] using ab initio pseudopotentials based on DFT with the exchange-correlation functional of Ceperley and Alder as parameterized by Perdw and Zunger (LDA-CAPZ).[12] All the possible structures concerned are opti- 
mized by the BFGS algorithm (proposed by Broyden, Fletcher, Goldfarb, and Shannon), [13] which provides a fast way of finding the lowest energy structure and supports cell optimization in the CASTEP code. The interaction between the ions and the valence electrons is described by using Vanderbilt's supersoft pseudopotential[14]. We used a plane wave cutoff of $310 \mathrm{eV}$ and a Brillouin zone sampling grid spacing of $2 \pi \times 0.04 \AA^{-1}$. Pseudoatomic calculations are performed for $\mathrm{N} 2 s^{2} 2 p^{3}$ and $\operatorname{Re} 5 s^{2} 5 p^{6} 5 d^{5} 6 s^{2}$. In the geometrical optimization, all forces on atoms are converged to less than $0.002 \mathrm{eV} / \AA$, all the stress components are less than $0.02 \mathrm{GPa}$, and the tolerance in self-consistent field (SCF) calculation is $5.0 \times 10^{-7} \mathrm{eV} /$ atom. Relaxation of the internal degrees of freedom is performed at each unit cell compression or expansion.

The standard method is used to calculate the elastic constants, in which the second derivatives of the internal energy of a crystal are determined as a function of properly chosen lattice distortions describing strains. In practice, by applying small elastic strains, the elastic constants are obtained from the change of energy or stress. Elastic moduli are given by using the elastic constants according to the Voigt-Reuss-Hill (VRH) approximation. [15] The Hill average, in general, is selected as the estimation of bulk modulus and shear modulus.

\section{RESULTS AND DISCUSSIONS}

\section{A. Structural property}

To search for the most stable structure of $\mathrm{ReN}_{2}$, we consider monoclinic, orthorhombic, tetrahedral, hexagonal, and cubic structures with a Re:N stoichiometry of 1:2, including these initial structures of $\mathrm{AuTe}_{2}\left(C 2 / m\right.$, No. 12), $\mathrm{CoSb}_{2}\left(P 2_{1} / c\right.$, No. 14), $\mathrm{GeS}_{2}(F d d 2$, No. 43), $\mathrm{CaCl}_{2}$ (Pnnm, No. 58), $\mathrm{OsB}_{2}$ (Pmmn, No. 59), $\beta-\mathrm{ReO}_{2}$ (Pbcn, No. 60), Pbca (8c Wycknoff sites, No. 61), Pnma (4c Wycknoff sites, No. 62), ReSi 2 (Immm, No. 71), $\mathrm{SiS}_{2}$ (Ibam, No. 72), $\mathrm{Cu}_{2} \mathrm{Sb}\left(P 4 / n m m\right.$, No. 129), rutile (P4 $2 / m n m$, No. 136), $\mathrm{MoSi}_{2}(I 4 / m m m$, No. 139), $P \overline{3} m 1$ (1 $a$ and $2 d$ Wycknoff sites, No 164), $\mathrm{CrSi}_{2}\left(P 6_{2} 22\right.$, No. 180), Fe 2 P (P $\overline{6} 2 m$,

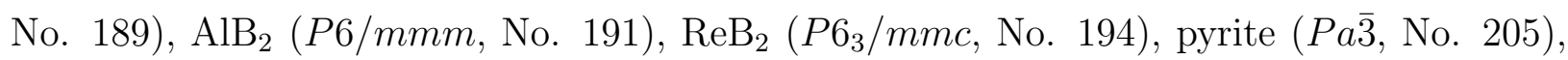
fluorite $\left(F m \overline{3} m\right.$, No. 225), and $\mathrm{NiTi}_{2}(F d \overline{3} m$, No. 227). Combining total energy with elastic constants analysis, four competing structures, i.e., Pbcn, P4 $4_{2} / m n m, P m m n$, and $F m \overline{3} m$, satisfy the mechanical stability critetion, [16, 17] in which the latter three phases 
have been discussed by Zhao et al.[10] The equilibrium volume $V_{0}$ and bulk modulus $\mathrm{B}_{0}$ are determined by fitting the total energy as a function of volume to the 3rd-order Birch Murnaghan equation of state (EOS).[18] Our calculated equilibrium parameters (see Table I) show that the rutile $\left(\mathrm{P}_{2} / \mathrm{mnm}\right)$ and the $\mathrm{Pbcn}$ phases have larger equilibrium volumes than the fluorite $(F m \overline{3} m)$ phase. From the calculated relative enthalpy $\Delta H$, one can see that the Pbcn phase is the most stable structure at zero pressure. In the optimized Pbcn structure, four rhenium atoms occupy $4 c$ Wycknoff sites $(y=0.1191)$ and eight $\mathrm{N}$ atoms hold $8 d$ sites $(x=0.2663, y=0.3526, z=0.0871)$. The nearest distance between $\operatorname{Re}(\mathrm{N})$ and $\mathrm{N}$ atoms is

1.955 (2.644) A. The orthorhombic Pbcn structure possesses a structure characterized by zigzag chains of Re atoms propagating along the $c$ axis of the unit cell with bond length and bond angle of $2.794 \AA$ and $122.4^{0}$, respectively. In order to determine the structural phase transition between competing structures, the enthalpy $H$ of each phase of $\mathrm{ReN}_{2}$ is computed. The enthalpy difference per formula unit (f.u.) as a function of pressure is plotted in Fig. 1. We do not observe structural phase transition from Pbcn phase to others up to $100 \mathrm{GPa}$. It is interesting to notice that Pbcn structure has been observed in experiment being the ground state of $\mathrm{ReO}_{2} \cdot[19]$

VED is defined as the total number of valence electron divided by volume per formula unit, which is an important factor when searching the superhard materials. The valence electron shell of Re is $6 \mathrm{~s}^{2}$ and $5 \mathrm{~d}^{5}$, and that of $\mathrm{N}$ is $2 \mathrm{~s}^{2} \mathrm{p}^{3}$ so that the total number of valence electrons is 17 for $\mathrm{ReN}_{2}$. VED obtained in four phases (Table I) is slightly lower than that of Re metal (0.4761 electrons $\left./ \AA^{3}\right)$ due to the adding of $\mathrm{N}$ atoms. High VED is good to resist the fracture, which contributes to high bulk pressure of $\mathrm{ReN}_{2}$ (Table I), predicting that $\mathrm{ReN}_{2}$ could be considered as a candidate of a superhard material.

\section{B. Elastic property}

The elastic constants of Pbcn structure obtained from our calculations $\left(c_{11}=365 \mathrm{GPa}\right.$, $c_{22}=553 \mathrm{GPa}, c_{33}=610 \mathrm{GPa}, c_{44}=138 \mathrm{GPa}, c_{55}=230 \mathrm{GPa}, c_{66}=83 \mathrm{GPa}, c_{12}=307 \mathrm{GPa}$, $\left.c_{13}=232 \mathrm{GPa}, c_{23}=242 \mathrm{GPa}\right)$ satisfy the mechanical stability requirement well, implying that the orthorhombic Pbcn structure is mechanically stable. As is concluded above, $\mathrm{ReN}_{2}$ has a very high bulk modulus, and therefore can be regarded as a candidate of a superhard material. However, a high bulk modulus by itself does not directly lead to high hardness. In 
general, both high bulk modulus and high shear modulus are indispensable to high hardness. Therefore, the shear modulus must be taken into account when searching for new superhard materials. The bulk modulus represents the resistance to fracture, while the shear modulus represents the resistance to plastic deformation. Moreover, two other factors are important for technological and engineering applications: Young's modulus $E$ and Poisson's ratio $\nu$. Young's modulus, defined as the ratio between stress and strain, is used to provide a measure of stiffness of the solid. The larger the value of $E$, the stiffer the material. The Young's modulus and Posson's ratio for an isotropic material are given by $E=\frac{9 B G}{3 B+G}, \nu=\frac{3 B-2 G}{2(3 B+G)}$, respectively,[15] where B and G represent Hill average bulk modulus and shear modulus. The calculated bulk modulus $B$, shear modulus $G$, and Young's modulus $E$ of $\mathrm{ReN}_{2}$ with Pbcn structure are $334 \mathrm{GPa}, 127 \mathrm{GPa}$, and $337 \mathrm{GPa}$, respectively, of which the bulk modulus of $\mathrm{ReN}_{2}$ is slightly smaller than that of Re metal (360 GPa). It is worthy to note that the bulk modulus obtained from the elastic constants agrees well with the one obtained through the fit to the 3rd-order Birch Murnaghan EOS $\left(\mathrm{B}_{0}\right)$, providing a consistent estimation of the compressibility for $\mathrm{ReN}_{2}$. Possion's ratio $\nu$ reflects the stability of a crystal against shear. This ratio can formally take values between -1 and 0.5 , which corresponds respectively to the lower limit where the material does not change its shape, or to the upper limit when the volume remains unchanged. Poisson's ratio of Pbcn structure is 0.3319, which is larger than 0.3 , indicating that there exists small volume change during elastic deformation. Also Possion's ratio provides more information on the characteristics of the bonding forces. It has been proved that $\nu=0.25$ is the lower limit for central-force solids and 0.5 is the upper limit, which corresponds to infinite elastic anisotropy. The high value $\nu$ for $\mathrm{ReN}_{2}$ means that the central interatomic forces are central.[20] In order to predict the brittle and ductile behavior of solids, Pugh introduced the ratio of the bulk modulus to shear modulus of polycrystalline phases. 21] A high (low) $B / G$ value is associated with ductility (brittleness). The critical value which separates ductile and brittle materials is about 1.75 . The significant difference obtained between bulk and shear modulus indicates the higher ductility of $\mathrm{ReN}_{2}$.

Now, we turn to discuss the elastic anisotropy of $\mathrm{ReN}_{2}$, which is estimated by the universal anisotropy index $A=5 \frac{G^{V}}{G^{R}}+\frac{B^{V}}{B^{R}}-6$. [22] The discrepancy of $A$ from zero determines the extent of single crystal anisotropy and accounts for both the shear and the bulk contributions. The obtained $A$ value is 1.252 , indicating that $\mathrm{ReN}_{2}$ has a large elastic anisotropy. Additionally, fractional axis compression as a function of pressure are plotted in Fig. 2. The curves 
presented in this figure clearly exhibit that: a bigger difference in fractional axis compression values exists for Pbcn phase, implicating that there is a evident structure deformation with increasing pressure; there is a transition point for compression along $b$ - and $c$-axis with increasing pressure, that is, $b$-axis is more difficult to be compressed than $c$-axis at 38 GPa above. This abnormality for compression along different axis can foretell that there is a structural phase transition at about 40 GPa. Further analysis of the change of the structural parameters (atomic coordination, bond length, et al.) shows that from 40 to 45 GPa fractional coordinations $x$ and $z$ in $8 d(x, y, z)$ sites (N atoms) change much, yielding the great change of the nearest distance between $\mathrm{N}$ atoms $\left(d_{N-N}\right)$. In detail, at $40 \mathrm{GPa}, x$ and $z$ are 0.2184 and 0.0752 , respectively. However, $x$ and $z$ reduce to 0.1385 and 0.0349 at $45 \mathrm{GPa}$, respectively. Correspondingly, the $d_{N-N}$ reduces from $2.359 \AA$ at $40 \mathrm{GPa}$ to 1.432 $\AA$ at $45 \mathrm{GPa}$.

Further, the Debye temperature $\Theta_{D}$ which correlates with many physical properties of materials, such as specific heat, elastic constants, and melting temperature is given. The Debye temperature $\Theta_{D}$ can be calculated by the equation $\Theta_{D}=\frac{h}{k}\left[\frac{3 n}{4 \pi}\left(\frac{\rho N_{A}}{M}\right)\right]^{1 / 3}\left[\frac{2}{3}\left(\frac{\rho}{G}\right)^{3 / 2}+\right.$ $\left.\frac{1}{3}\left(\frac{\rho}{B+4 G / 3}\right)^{3 / 2}\right]^{-1 / 3},[23]$ where $h$ is Plank's constant, $k$ is Boltzmann's constant, $N_{A}$ is Avogadro's number, $\rho$ is density, $M$ is the molecular weight and $n$ is the number of atoms in the molecule. The calculated $\Theta_{D}$ value of $735 \mathrm{~K}$ in $\mathrm{Pbcn}$ is comparative to the values of $866.4 \mathrm{~K}$ in $\mathrm{ReB}_{2}$ [24], $850 \mathrm{~K}$ in $\mathrm{ReO}_{2}[19]$ and $691 K$ in $\mathrm{OsN}_{2} \cdot[6]$ Employing the correlation between the shear modulus and Vickers hardness reported by Teter [25] for a wide variety of hard materials, the indentation hardness of $\mathrm{ReN}_{2}$ can be estimated to be approximately $17.1 \mathrm{GPa}$ , which is comparable to that of $\mathrm{Si}_{3} \mathrm{~N}_{4}(21 \pm 3 \mathrm{GPa}) .[25]$ It is well known that the hardness of superhard materials should be higher than $40 \mathrm{GPa}$, indicating that $\mathrm{ReN}_{2}$ is not a superhard material. Jhi et al [26] proposed that the hardness is determined by elastic constant $c_{44}$ rather than shear modulus. In our case, we note that there is little difference between the $c_{44}$ value of elastic constant and shear modulus. Accordingly, both Teter estimation and Jhi's viewpoint of hardness actually achieve a consistent result for $\mathrm{ReN}_{2}$.

\section{Electronic property}

In order to understand the nature of elastic property, the electronic density of states is also analyzed. The total and partial density of states (DOS) with respect to Fermi level 
is shown in Fig. 3. Finite DOS at Fermi level $N\left(\mathrm{E}_{F}\right)$ of 5.035 states/eV shows that $\operatorname{ReN}_{2}$ is metallic. The electrons from Re- $5 d$ and the N-2p states both contribute much to the DOS near the Fermi level. That is, the electronic structure of $\mathrm{ReN}_{2}$ is governed by strong hybridization between the Re- $5 d$ and N-2p states, imaging that there is a covalent bond in between Re and $\mathrm{N}$ atoms. The existence of covalent bonding contributes to large bulk modulus, i.e., strong incompressibility. However, we have not found any pseudogap that

observed in $\mathrm{ReB}_{2},\left[24\right.$, 27] $\mathrm{OsB}_{2}[28]$ and $\mathrm{OsN}_{2}$, [6] implying that covalent bonding in between Re and $\mathrm{N}$ atoms is not so strong. Besides, the calculated net charges on $\mathrm{N}$ and Re atom are -0.57 e and 1.15 e, which indicates that the chemical bonding between Re and $\mathrm{N}$ have some characteristics of ionicity. The occurrence of ionic bonding results in the smaller shear modulus, which explains the reason of the not so large hardness in $\mathrm{ReN}_{2}$.

\section{CONCLUSION}

In conclusion, the most stable structure of $\mathrm{ReN}_{2}$ is explored based on first-principles methods in the framework of density functional theory within local density approximation. It results that orthorhombic Pbcn structure is the most stable structure at ambient pressure. $\mathrm{ReN}_{2}$ is a metallic, super-incompressible solid and presents large elastic anisotropy. The estimated hardness is comparative to that of $\mathrm{Si}_{3} \mathrm{~N}_{4}$. The structural determination and the discussion of the elastic and electronic properties may be of use in the synthesis and high pressure study of $\mathrm{ReN}_{2}$.

\section{ACKNOWLEDGEMENT}

This work was supported by the special Funds for Major State Basic Research Project of China(973) under grant no. 2007CB925004, 863 Project, Knowledge Innovation Program of Chinese Academy of Sciences, and Director Grants of CASHIPS. Part of the calculations were performed in Center for Computational Science of CASHIPS and the Shanghai 
Supercomputer Center.

[1] J. C. Crowhurst, A. F. Goncharov, B. Sadigh, C. L. Evans, P. G. Morrall, J. L. Ferreira, and A. G. Nelson, Science 311 (2006) 1275.

[2] R. B. Kaner, J. J. Gilman and S. H. Tolbert. Science 308 (2006) 1268.

[3] H-y. Chung, M. B. Weinberger, J. B. Levine, A. Kavner, J-M. Yang, S. H. Tolbert, and R. B. Kaner, Science 316 (2007) 436.

[4] A. F. Young, C. Sanloup, E. Gregoryanz, S. Scandolo, R. J. Hemley, and H. K. Mao, Phys. Rev. Lett. 96 (2006) 155501.

[5] A. F. Young, J. A. Montoya, C. Sanloup, M. Lazzeri, E. Gregoryanz, and S. Scandolo, Phys. Rev. B 73 (2006) 153102.

[6] Z. Wu, X. Hao, X. Liu, and J. Meng, Phys. Rev. B 75 (2007) 054115.

[7] Z. W. Chen, X. J. Guo, Z. Y. Liu, M. Z. Ma, Q. Jing, G. Li, X. Y. Zhang, L. X. Li, Q. Wang, Y. J. Tian, and R. P. Liu, Phys. Rev. B 75 (2007) 054103.

[8] R. Yu, Q. Zhan, and L-C. De Jonghe, Angew. Chem. Int. Ed. 46 (2007) 1136.

[9] S. La Placa and B. Post, Acta Crystallogr. 15 (1962) 97.

[10] E. Zhao and Z. Wu, Comput. Mat. Sci. 44 (2008) 531.

[11] M. D. Segall, P. J. D. Lindan, M. J. Probert, C. J. Pickard, P. J. Hasnip, S. J. Clark, M. C. Payne, J. Phys.: Cond. Matt. 14 (2002) 2717.

[12] D. M. Ceperley and B. J. Alder, Phys. Rev. Lett. 45566 (1980); J. P. Perdew and Y. Wang, Phys. Rev. B 45 (1992) 13244 .

[13] B. G. Pfrommer, M. Cote, S. G. Louie, and M. L. Cohen, J. Comput. Phys. 131 (1997) 133.

[14] D. Vanderbilt, Phys. Rev. B 41 (1990) R7892.

[15] R. Hill, Proc. Phys. Soc. London 65 (1952) 349.

[16] O. Beckstein, J. E. Klepeis, G. L. W. Hart, and O. Pankratov, Phys. Rev. B 63 (2001) 134112.

[17] M. Born and K. Huang, Dynamical Theory of Crystal Lattices, Clarebdom, Oxford, 1956.

[18] J. -P. Poirier, Introduction to the physics of the Earth's Interior, Cambridge University Press, Cambridge, 2000.

[19] Y. L. Li and Z. Zeng, Chin. Phys. Lett 25 (2008) 4086.

[20] P. Ravindran, L. Fast, P. A. Korzhavyi, B. Johansson, J. Wills, and O. Eriksson, J. Appl. 
Phys. 84 (1998) 4891.

[21] S. F. Pugh, Philos. Mag. 45 (1954) 823.

[22] S. I. Ranganathan and M. Ostoja-Starzewski, Phys. Rev. Lett. 101 (2008) 055504.

[23] O. L. Anderson, J. Phys. Chem. Solids 24 (1963) 909.

[24] X. Hao, Y. Xu, Z. Wu, D. Zhou, X. Liu, X. Cao, and J. Meng, Phys. Rev. B 74 (2006) 224112.

[25] D. M. Teter, MRS. Bull. 23 (1998) 22.

[26] S-H. Jhi, J. Ihm, S. G. Louie, and M. L. Cohen, Nature 399 (1999) 132.

[27] Y. L. Li, G. H. Zhong, and Z. Zeng, accepted by Chin. Phys. B (2009)

[28] H. Y. Gou, L. Hou, J. W. Zhang, H. Li, G. F. Sun, and F. M. Gao, Appl. Phys. Lett. 88 (2006) 221904. 


\section{TABLE CAPTIONS}

TABLE I: Equilibrium lattice parameters, $V_{0}\left(\AA^{3}\right), a(\AA), b(\AA), c(\AA)$, density $\rho\left(\mathrm{g} / \mathrm{cm}^{3}\right)$, valence-electron density $\rho_{e}$ (electrons $\left./ \AA^{3}\right)$, bulk modulus $B_{0}(\mathrm{GPa})$, and enthalpy difference per chemical formula unit (f.u.) $\triangle \mathrm{H}(\mathrm{eV}) . V_{0}$ is of per chemical f.u. 


\section{FIGURE CAPTIONS}

Fig.1: Enthalpy difference per formula unit as a function of pressure. $P 4_{2} / \mathrm{mnm}$ is taken as a reference point.

Fig.2: Fractional axis compression as a function of pressure for $P b c n$ structure.

Fig.3: Total and partial density of states for Pbcn structure. Vertical dotted line indicates the Fermi level. 
TABLE I: Li et al.

\begin{tabular}{|c|c|c|c|c|c|}
\hline & Pbcn & $P 4_{2} / m n m$ & $F m \overline{3} m$ & Pmmn & Reference \\
\hline \multirow[t]{2}{*}{$V_{0}$} & 31.251 & 31.693 & 27.712 & 26.779 & This work \\
\hline & & 31.7 & 27.7 & 26.7 & {$[10]$} \\
\hline \multirow[t]{2}{*}{$a$} & 4.518 & 4.769 & 4.801 & 8.088 & This work \\
\hline & & 4.775 & 4.803 & 8.083 & {$[10]$} \\
\hline \multirow[t]{2}{*}{$b$} & 5.657 & & & 2.380 & This work \\
\hline & & & & 2.385 & [10] \\
\hline \multirow[t]{2}{*}{$c$} & 4.902 & 2.789 & & 2.782 & This work \\
\hline & & 2.78 & & 2.77 & {$[10]$} \\
\hline$\rho$ & 11.385 & 11.217 & 12.838 & 12.406 & This work \\
\hline$\rho_{e}$ & 0.416 & 0.410 & 0.469 & 0.453 & This work \\
\hline $\mathrm{B}_{0}$ & 331.3 & 337.8 & 381.9 & 365.2 & This work \\
\hline$\triangle \mathrm{H}$ & 0 & 0.015 & 1.457 & 1.663 & This work \\
\hline
\end{tabular}




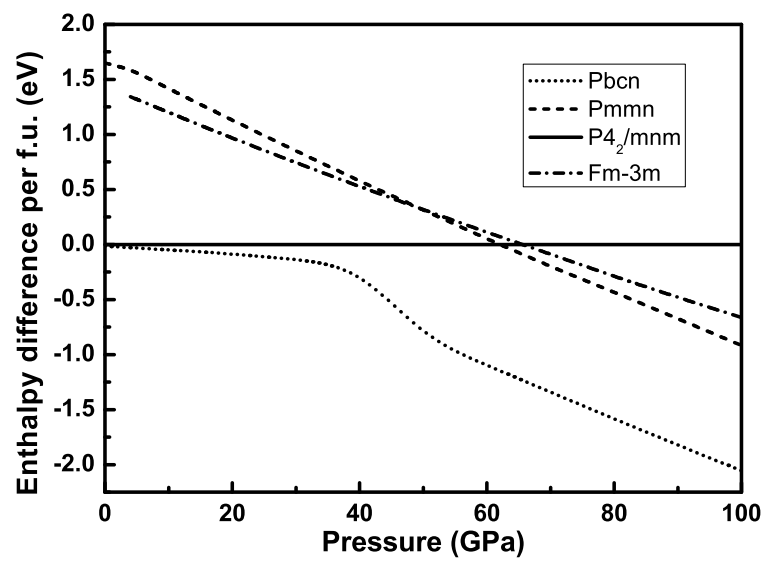

FIG. 1: Li et al. 


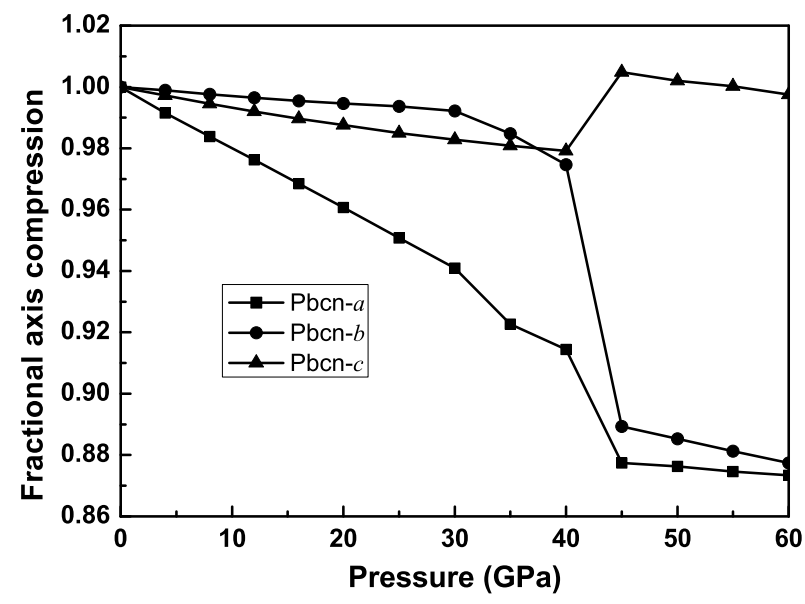

FIG. 2: Li et al. 


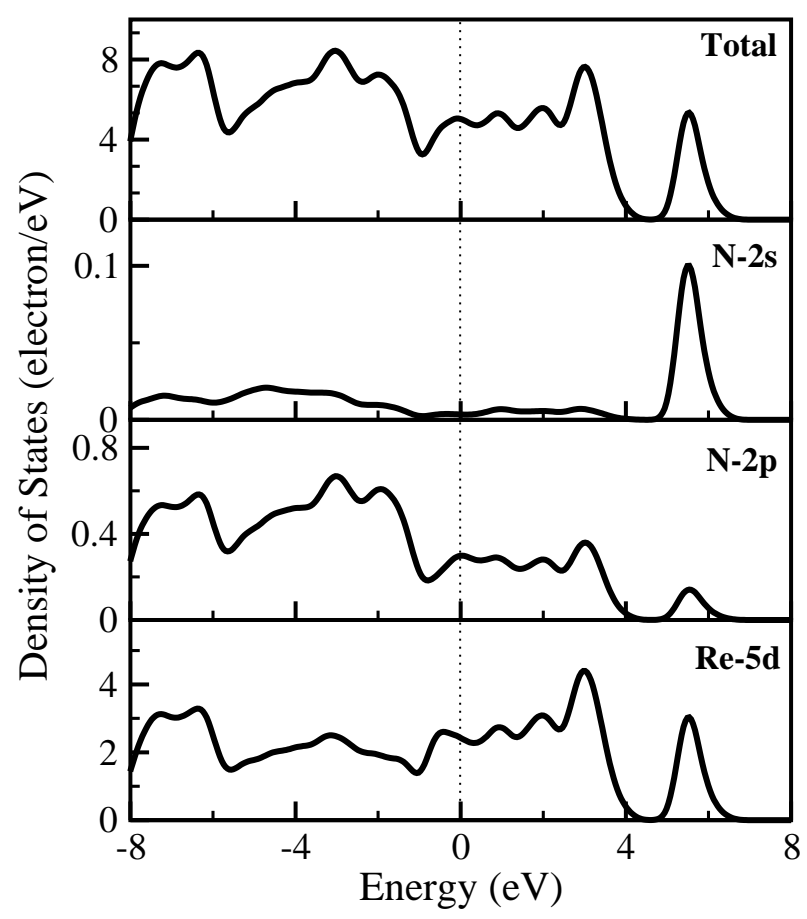

FIG. 3: Li et al. 